\title{
Predicting Fatigue Crack Initiation Life of Plate-like Component by Damage Mechanics and Finite Element Method
}

\author{
Wen-Feng TAN ${ }^{1, a,{ }^{*}}$, Yan-Shen YANG ${ }^{1}$ \\ ${ }^{1}$ Key Laboratory of Mechanical Reliability for Heavy Equipments and Large Structures of Hebei \\ Province, Yanshan University, Qinhuangdao 066004, China \\ atwfysu@163.com \\ ${ }^{*}$ Corresponding author
}

Keywords: Damage Mechanic, Finite Element Method, Additional Load, Crack Initiation Life.

\begin{abstract}
Applied fatigue damage mechanics and finite element method to study the fatigue crack initiation life problems of component. Fatigue crack initiation life is obtained by using the method of additional load, just a stiffness matrix calculation, greatly reducing the workload of calculation. With the damage degree of the critical element partitioning step of the damage evolution, the damage degree increases unceasingly obtains the corresponding, corresponding to the stress field, strain field and the load matrix, and then to calculate the equivalent stress, the application of damage evolution equation for calculating fatigue crack forming life corresponding. Until the damage degree of the critical element to 1 so far, the fatigue crack initiation life is obtained by adding the fatigue crack initiation lives with different damage variable.
\end{abstract}

\section{Introduction}

The fatigue life calculation is an important problem in engineering. Fatigue failure is a process of time, each load cycle will cause the components of internal damage evolving, even in areas of stress concentration will generate fatigue crack. Component damage increases with load cycles, the stiffness will also along with the change of load cycles. In general, the process of stress analysis, every stiffness matrix after injury need to compute, it makes calculation workload is very large. In order to avoid repeated computation of the stiffness matrix, the literature [1] proposed a concept of additional load. Fatigue crack initiation life is obtained by using the method of additional load, just a stiffness matrix calculation, greatly reducing the workload of calculation.

Damage mechanics and finite element method is applied in this article, the widespread damage component fatigue crack initiation life estimate is studied. The calculate format of damage mechanics and finite element method is established. The calculation method and steps of fatigue crack formation life is given.

\section{Finite Element Scheme}

For linear elastic component, should satisfy the equilibrium differential equation and stress boundary conditions[2].

$$
\frac{\partial \sigma_{i j}}{\partial x_{j}}+f_{i}=0, \sigma_{i j} l_{j}=p_{i}
$$

where $\sigma_{i j}$ is variation of stress components, $f_{i}$ is variation of body force components, $p_{i}$ variation of surface components.

For components with damage, constitutive relations[2] can be expressed as follows:

$$
\sigma_{i j}=C_{i j k l}(1-D) \varepsilon_{k l}
$$


where $C_{i j k l}$ is elastic constants, $\varepsilon_{k l}$ is variation of strain components. $D$ is damage variable, For metal materials, $D$ can be regarded as a scalar, and, $0 \leq D \leq 1$.

Introducing variation of equivalent stress [3]

$$
\sigma_{e q}=\sqrt{\frac{3}{2} s_{i j} s_{i j}}
$$

where $s_{i j}$ is variation of partial stress tensor, $s_{i j}=\sigma_{i j}-\delta_{i j} \delta_{r s} \sigma_{r s}$. Suppos

$$
\sigma_{i j}^{E}=C_{i j k l} \varepsilon_{k l}, \quad \sigma_{i j}^{D}=-D C_{i j k l} \varepsilon_{k l}
$$

According to the type (2), to be seen

$$
\sigma_{i j}=\sigma_{i j}^{E}+\sigma_{i j}^{D}
$$

The substitution of Eqs.(5) into Eq.(1) yields

$$
\frac{\partial \sigma_{i j}^{E}}{\partial x_{j}}+f_{i}+f_{i}^{D}=0 \quad \sigma_{i j}^{E} l_{j}=p_{i}+p_{i}^{D}
$$

where $f_{i}^{D}$ is additional body force components, $p_{i}^{D}$ is additional surface force components, and respectively

$$
f_{i}^{D}=\frac{\partial \sigma_{i j}^{D}}{\partial x_{j}}, p_{i}^{D}=-\sigma_{i j}^{D} l_{j}
$$

For plane problems, the introduction of constant strain element, the element strain column matrix $\{\varepsilon\}_{e}$ can be expressed as a node displacement column matrix $\{\delta\}_{e}$

$$
\{\varepsilon\}_{e}=[B]_{e}\{\delta\}_{e}
$$

where $[B]_{e}$ is geometry matrix

The constitutive relation of damage coupling is

$$
\sigma_{e}=\left(1-D_{e}\right)[E]_{e}\{\varepsilon\}_{e}=\left(1-D_{e}\right)[E]_{e}[B]_{e}\{\delta\}_{e}
$$

where $D_{e}$ is element damage variable, $[E]_{e}$ is element elastic matrix. Or written

$$
\{\sigma\}_{e}=\{\sigma\}_{e}^{E}+\{\sigma\}_{e}^{D}
$$

where $\{\sigma\}_{e}$ is element stress matrix, $\{\sigma\}_{e}^{E}$ is element stress matrix of no damage body, $\{\sigma\}_{e}^{D}$ is element stress matrix of damage body

$$
\{\sigma\}_{e}^{E}=[E]_{e}[B]_{e}\{\delta\}_{e},\{\sigma\}_{e}^{D}=-D_{e}[E]_{e}[B]_{e}\{\delta\}_{e}
$$

Element nodal force column matrix $\{F\}_{e}$ by virtual work principle is expressed as nodal displacement array

$$
\{F\}_{e}=[k]_{e}\{\delta\}_{e}-\{F\}_{e}^{D}
$$

where $[k]_{e}$ is element stiffness matrix of no damage body 


$$
[k]_{e}=\int_{V_{e}}[B]_{e}^{T}[E]_{e}[B]_{e} \mathrm{~d} V
$$

and

$$
\{F\}_{e}^{D}=D_{e}[k]_{e}\{\delta\}_{e}
$$

According to the virtual work equation, the total load column matrix have to

$$
\{F\}_{e}^{D}=D_{e}[k]_{e}\{\delta\}_{e}
$$

Or written

$$
[K]\{\delta\}=\{F\}+\{F\}^{D}
$$

where $[K]$ is global stiffness matrix of no damage body, $\{F\}^{D}$ is additional load column matrix, and

$$
[K]=\left(\sum_{e}[A]_{e}^{T}[k]_{e}[A]_{e}\right) \quad\{F\}^{D}=\left(\sum_{e} D_{e}[A]_{e}^{T}[k]_{e}[A]_{e}\right)\{\delta\}
$$

where $[A]_{e}$ is transformation matrix.

Thus, given the damage field of displacement analysis problem, after the introduction of additional load column matrix $\{F\}^{D}$ can be converted into noninvasive displacement analysis problem. In this way, only to form a global stiffness matrix can be.

\section{Fatigue Crack Initiation Life Calculation}

The damage evolution equation is as follows[1]

$$
\frac{\mathrm{d} D}{\mathrm{~d} N}=a\left\{\frac{\sigma_{e q}}{1-D}\right\}^{m}
$$

where $N$ is stress cycle times that is life, $\alpha, m$ is material constants in the damage evolution equation

The fatigue crack initiation life of damage bodies is calculated using damage mechanics and finite element method. The formulas and calculation steps are as follows

(1) The displacement field $u_{i 0}$, strain field $\varepsilon_{i j 0}$ and stress field $\sigma_{i j 0}$ of no damage body are analyzed. Its equivalent stress maximum point is computed. We define it for dangerous point.

(2) Given the damage variable growth step of dangerous point $\Delta D_{0}(0)$. By Eq.(18) calculates the corresponding load cycle times and any point increase value of damage variable, respectively

$$
\Delta N_{0}=\frac{\Delta D_{0}(0)}{a\left\{\sigma_{e q 0}(0)\right\}^{m}}, \quad \Delta D_{0}(x)=\left\{\frac{\sigma_{e q 0}(x)}{\sigma_{e q 0}(0)}\right\}^{m} \Delta D_{0}(0)
$$

(3) Suppose

$$
D_{1}(0)=\Delta D_{0}(0), D_{1}(x)=\Delta D_{0}(x)
$$

And then the displacement field $u_{i 1}$, strain field ${ }^{\varepsilon_{i j 1}}$ and stress field ${ }^{\sigma_{i j 1}}$ are obtained by using the damage mechanics and finite element method.

(4) Given the damage variable growth step of dangerous point $\Delta D_{1}(0)$. By Eq.(18) calculates the corresponding load cycle times and any point increase value of damage variable, respectively 


$$
\Delta N_{1}=\frac{\Delta D_{1}(0)}{a\left\{\sigma_{e q 1}(0)\right\}^{m}\left\{1-D_{1}(0)\right\}^{-m}}, \Delta D_{1}(x)=\left\{\frac{\sigma_{e q 1}(x)}{\sigma_{e q 1}(0)}\right\}^{m}\left\{\frac{1-D_{1}(0)}{1-D_{1}(x)}\right\}^{-m} \Delta D_{1}(0)
$$

(5) Suppose

$$
D_{2}(0)=D_{1}(0)+\Delta D_{1}(0), D_{2}(x)=D_{1}(x)+\Delta D_{1}(x)
$$

And then the displacement field $u_{i 2}$, strain field $\varepsilon_{i j 2}$ and stress field $\sigma_{i j 2}$ are obtained by using the damage mechanics and finite element method.

(6) Given the damage variable growth step of dangerous point $\Delta D_{2}(0)$. By Eq.(18) calculates the corresponding load cycle times and any point increase value of damage variable, respectively

$$
\Delta N_{2}=\frac{\Delta D_{2}(0)}{a\left\{\sigma_{e q 2}(0)\right\}^{m}\left\{1-D_{2}(0)\right\}^{-m}} \quad \Delta D_{2}(x)=\left\{\frac{\sigma_{e q 2}(x)}{\sigma_{e q 2}(0)}\right\}^{m}\left\{\frac{1-D_{2}(0)}{1-D_{2}(x)}\right\}^{-m} \Delta D_{2}(0)
$$

(7) And so on, the damage variable is

$$
D_{n}(0)=D_{n-1}(0)+\Delta D_{n-1}(0), D_{n}(x)=D_{n-1}(x)+\Delta D_{n-1}(x)
$$

The displacement field $u_{i n}$, strain field ${ }^{\varepsilon_{i j n}}$ and stress field ${ }^{\sigma_{i j n}}$ are obtained by using the damage mechanics and finite element method.

(8) Given the damage variable growth step of dangerous point $\Delta D_{n}(0)$. By Eq.(18) calculates the corresponding load cycle times and any point increase value of damage variable, respectively

$$
\Delta N_{n}=\frac{\Delta D_{n}(0)}{a\left\{\sigma_{e q n}(0)\right\}^{m}\left\{1-D_{n}(0)\right\}^{-m}}, \Delta D_{n}(x)=\left\{\frac{\sigma_{e q n}(x)}{\sigma_{e q n}(0)}\right\}^{m}\left\{\frac{1-D_{n}(0)}{1-D_{n}(x)}\right\}^{-m} \Delta D_{n}(0)
$$

(9) The above steps to $D_{k}(0)=1$ so far. The sum of the corresponding load cycle times are the damage fatigue crack initiation life $N$.

$$
N=\sum_{n=0}^{k} \Delta N_{n}
$$

\section{Conclusion}

(1) The calculate format of damage mechanics and finite element method is established.

(2) Presents the application of damage mechanics and finite element method to calculate the damage body fatigue crack formation life method and steps.

(3) This method can calculate fatigue crack initiation life of component, also can calculate the fatigue crack propagation life of component.

\section{References}

[1]X. Zhang, J. Zhao, Applied Fatigue Damage Mechanics of Metallic Structural Members, National Defence Industry Press, China 1998.

[2]Z. Zhuang, C. P. Jiang, Engineering fracture and damage, Mechanical Industry Press, China 2004.

[3]B. Y. Yang, Y. T. Zhang, Engineering elastic-plastic mechanics, Mechanical Industry Press, China 2003. 